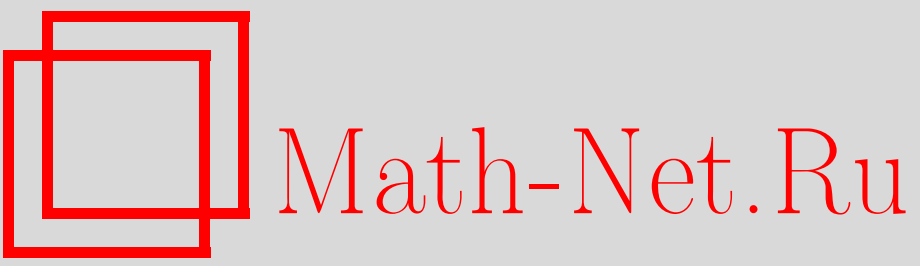

В. П. Бурский, Обобщенные решения граничных задач для дифференциальных уравнений общего вида, УМН, 1998, том 53, выпуск 4, 215-216

DOI: https://doi.org/10.4213/rm61

Использование Общероссийского математического портала Math-Net.Ru подразумевает, что вы прочитали и согласны с пользовательским соглашением

http://www.mathnet.ru/rus/agreement

Параметры загрузки:

IP: 3.80 .253 .173

26 апреля 2023 г., 17:59:34 


\title{
ОБОБШЕННЫЕ РЕШЕНИЯ ГРАНИЧНЫХ ЗАДАЧ ДЛЯ ДИФФЕРЕНЦИАЛЬНЫХ УРАВНЕНИЙ ОБЩЕГО ВИДА
}

\author{
В. П. БУРСКИй
}

Пусть $\mathscr{L}=\sum_{|\alpha| \leqslant l} a_{\alpha}(x) D^{\alpha}, a_{\alpha} \in C^{\infty}(\bar{\Omega}), D^{\alpha}=\frac{(-i \partial)^{|\alpha|}}{\partial x^{\alpha}}-$ диффференциальньй оператор общего вида с $(j \times k)$-матрицами $a_{\alpha}$, элементами которых являются гладкие комплекснозначные функции, и пусть $\Omega$ - произвольная область в $\mathbb{R}^{n}$. Оператор $\mathscr{L}$ порождает формально сопряженный оператор $\mathscr{L}^{+}=\sum_{|\alpha| \leqslant l} D^{\alpha}\left(a_{\alpha}^{*}(x) \cdot\right)$, где $a_{\alpha}^{*}(x)$ - сопряженная матрица.

Ниже будут рассмотрены граничные задачи в обобщенной постановке для уравнений вида:

$$
\mathscr{L}^{+} \circ A \circ \mathscr{L} u=f
$$

с некоторым непрерьвным (вообще говоря, нелинейным) оператором $A: L_{2}^{k}(\Omega) \rightarrow L_{2}^{k}(\Omega)$ и их связь с теорией расширений операторов [1].

Минимальный оператор $L_{0}$, определяемьй как замшкание оператора $\mathscr{L}$, первоначально заданного на $C_{0}^{\infty}(\Omega)$, в норме грабфика $\|u\|_{L}^{2}=\|u\|_{L_{2}^{j}(\Omega)}^{2}+\|\mathscr{L} u\|_{L_{2}^{k}(\Omega)}^{2}$ и аналогичньй минималњный оператор $L_{0}^{+}$порождают максимальные операторы $L=\left(L_{0}^{+}\right)^{*}, L^{+}=L_{0}^{*}$ с помощью сопряжения в гильбертовых пространствах. Области определения $D\left(L_{0}\right), D\left(L_{0}^{+}\right), D(L), D\left(L^{+}\right)$ этих операторов являются гильбертовыми пространствами в соответствующей норме графика. Будем для простоты считать, что каждая функция из $D(L)$ или $D\left(L^{+}\right)$может быть приближена гладкими функциями в соответствующей норме графика. Введем (см. [2]) пространство Коши $C(L)=D(L) / D\left(L_{0}\right)$, а также фактор-отображение $\Gamma: D(L) \rightarrow C(L)$ и аналогично $C\left(L^{+}\right)$и $\Gamma^{+}$.

Рассмотрим следующие условия:

$$
\begin{aligned}
& \text { оператор } L_{0}: D\left(L_{0}\right) \rightarrow L_{2}^{k}(\Omega) \text { имеет непрерьвный левый обратньй; } \\
& \text { оператор } L_{0}^{+}: D\left(L_{0}^{+}\right) \rightarrow L_{2}^{j}(\Omega) \text { имеет непрерьвный левый обратньй. }
\end{aligned}
$$

Однородной граничной задачей (см. [2]) назьвается задача нахождения решения соотношений

$$
L u=f, \quad \Gamma u \in B,
$$

где $B$ - линейное подпространство в пространстве Коши, определяющее граничную задачу. Задача (4) называется корректной, если оператор $L_{B}=\left.L\right|_{D\left(L_{B}\right)}, D\left(L_{B}\right)=\Gamma^{-1} B$, является разрешимым расширением оператора $L_{0}$, т.е. если оператор $L_{B}: D\left(L_{B}\right) \rightarrow L_{2}^{k}(\Omega)$ имеет непрерывньй обратньй (который является правьм обратным к $L$ ). Хорошо известно (М. И. Вишик [1], в интерпретации Л. Хермандера [2]), что у оператора $L_{0}$ сушествует разрешимое расширение (и для оператора $L$ существует корректная граничная задача $(4))$ тогда и только тогда, когда выполнены условия (2) и (3). Сопряженной к (4) назьвается граничная задача: $L^{+} v=g$, $\Gamma^{+} v \in B^{+}$, где пространство $B^{+}=\Gamma^{+} D_{B}^{+}, D_{B}^{+}=\left\{v \in D\left(L^{+}\right) \mid \forall u \in \Gamma^{-1}(B),[u, v]=0\right\}$, порождено формой Грина: $[u, v]=\int_{\Omega}\left(L u \cdot \bar{v}-u \cdot \overline{L^{+} v}\right) d x=\left\langle\mathscr{L}_{\partial \Omega} \Gamma u, \Gamma^{+} v\right\rangle=-\left\langle\Gamma u, \mathscr{L}_{\partial \Omega}^{+} \Gamma^{+} v\right\rangle$.

Рассмотрим теперь обобщенные постановки граничных задач (cp. [3], [4]). Задача (4) порождает следующую граничную задачу для уравнения (1): найти функцию $u \in D\left(L_{B}\right)$, назьваемую обобщенным решением, такую, что для любой $v \in D\left(L_{B}\right)$ выполнено интегральное тождество

$$
\left\langle A \circ L_{B} u, L_{B} v\right\rangle=\langle f, v\rangle, \quad f \in D^{\prime}\left(L_{B}\right),
$$

которое можно понимать как задачу решения уравнения $Q u: L_{B}^{\prime} \circ A \circ L_{B} u=f$, где $L_{B}^{\prime}: L_{2}^{k}(\Omega) \rightarrow$ $D^{\prime}\left(L_{B}\right)$ - дуальный оператор. Если функция $u$ такова, что $L u \in D\left(L^{+}\right)$, то граничное условие здесь можно записать в виде: $\Gamma u \in B ; \Gamma^{+} A \mathscr{L} u \in B^{+}$. Задачу (5) назовем задачей Дирихле, если $B=\{0\}$, и задачей Неймана, если $B=C(L)$. Задачу (5) назовем нормалшно корректно разрешимой, если пространство $\operatorname{Im} Q \subset D^{\prime}\left(L_{B}\right)$ совпадает с ортогональным пространством $K$ к ядру ker $L_{B}$ и существует непрерьвный оператор $R: K \rightarrow D\left(L_{B}\right)$, правьй обратный к оператору $Q: D\left(L_{B}\right) \rightarrow K$. Задачу (5) назовем корректно разрешимой, если оператор $Q: D\left(L_{B}\right) \rightarrow D^{\prime}\left(L_{B}\right)$ имеет непрерьвный двусторонний обратньй $Q^{-1}$. 
УТВЕРЖДЕНИЕ 1. Задача (5) нормально корректно разрешима тогда и только тогда, когда оператор $L_{B}$ нормально разрешим и оператор $P \circ A$, где $P: L_{2}^{k}(\Omega) \rightarrow \operatorname{Im} L_{B}$ - ортопроектор, является гомеоморфизмом пространства $\operatorname{Im} L_{B}$ на себя. Задача (5) корректно разрешима тогда и только тогда, когда она нормально корректно разрешима и ядро $\operatorname{ker} L_{B}$ тривиально.

Из утверждения 1 вытекают полезные следствия.

УТВеРЖДЕНИЕ 2. Пусть расширение $L_{B}$ разрешимо. Тогда задача (5) корректно разрешима, если и только если оператор $А$ является гомеоморфизмом в пространстве $L_{2}^{k}(\Omega)$. Если $A=\mathrm{id}$, то корректность задачи (4) влечет корректную разрешимость задачи (5).

УтВеРЖДЕнИЕ 3. Пусть $A=\mathrm{id}$. Задача Дирихле (5) $\left(\right.$ с $\left.D\left(L_{B}\right)=D\left(L_{0}\right)\right)$ корректно разрешима тогда и только тогда, когда выполнено условие Вишика (2).

УтвеРжДЕНИЕ 4. Пусть $A=$ id. Задача Неймана (5) $\left(\right.$ с $\left.D\left(L_{B}\right)=D(L)\right)$ нормально корректно разрешима тогда и только тогда, когда оператор $L$ нормально разрешим, в частности, если выполнено условие Вишика (3) (в этом случае $\operatorname{Im} L=L_{2}^{k}(\Omega)$ ).

ПримеР 1. Рассмотрим обобщенную задачу Дирихле для уравнения Лапласа $\Delta u=f$ : $\mathscr{L}=\operatorname{grad}, \mathscr{L}^{+}=\operatorname{div}, D(L)=W_{2}^{1}(\Omega), D\left(L_{0}\right)=\stackrel{\circ}{W_{2}^{1}}(\Omega), f \in\left[\stackrel{\circ}{W_{2}^{1}}(\Omega)\right]^{\prime}$. Утверждение 3 говорит здесь, что обобщенная задача Дирихле корректно разрешима, если и только если в области $\Omega$ справедливо неравенство Фридрихса: $\|\nabla u\| \geqslant C\|u\| \forall u \in C_{0}^{\infty}(\Omega)$, которое является неравенством Вишика для оператора $\nabla$.

ПримеР 2. Рассмотрим обобщенную задачу Неймана для уравнения Лапласа. Из утверждения 4 вытекает, в частности, что эта задача нормалшно корректно разрешима в области $\Omega$ с конечномерньм пространством первых когомологий с коэффициентами в поле $\mathbb{C}$ (например, в ограниченной области с гладкой границей). Действительно, в этом случае по теореме де Рама замкнутое в $L_{2}^{k}(\Omega)$ ядро непрерьвного оператора $\operatorname{rot}(=$ внешний дифференциал) шире образа оператора grad на конечномерное пространство, поэтому пространство потенциальных векторных полей $\nabla W_{2}^{1}(\Omega)$ замкнуто в $L_{2}^{k}(\Omega)$.

ПримеР 3. Рассмотрим обобщенную задачу (5) для уравнения (1) со скалярными функциями и операторами, где $A: L_{2}(\Omega) \rightarrow L_{2}(\Omega)$ - оператор Немыцкого, заданный отображением $a$ в $\mathbb{R}: A(v)(x)=a(v(x))$. Условия: 1$)$ гомеоморфность отображения $a: \mathbb{R} \rightarrow \mathbb{R}$ и 2$)$ линейный рост $C_{1}|\xi|+C_{2} \leqslant a(\xi) \leqslant C_{3}|\xi|+C_{4}$ обеспечивают гомеоморфиность отображения $A$, поэтому корректность задачи (4) по утверждению 2 влечет корректную разрешимость нелинейной задачи (5) с таким оператором $A$. В частности, отсюда следует корректная разрешимость следующей граничной задачи: $\square a(\square u)=f \in D^{\prime}\left(\square_{B}\right)$ в единичном круге $K, u \in D\left(\square_{B}\right), a(\square u) \in D\left(\square_{B^{+}}\right)$, $\square=\frac{\partial^{2}}{\partial x_{1} \partial x_{2}}$, где $D\left(\square_{B}\right)=\left\{u \in D(\square) \mid u(\tau)=0\right.$ на $\Gamma_{1}=\left\{\angle \tau \in \partial K, \frac{\pi}{2} \leqslant \tau \leqslant 2 \pi\right\}$, $u_{\nu}^{\prime}(\tau)=0$ на $\left.\Gamma_{2}=\left\{\angle \tau \in \partial K, \pi \leqslant \tau \leqslant \frac{3 \pi}{2}\right\}\right\}, u(\tau) \in B ; D\left(\square{ }_{B}^{+}\right)=\left\{u \in D(\square) \mid \Gamma^{+} u \in B^{+}\right\}$, $B^{+}=\left\{u(\tau) \mid u(\tau)=0\right.$ на СГ $1=\left\{\angle \tau \in \partial K, 0 \leqslant \tau \leqslant \frac{\pi}{2}\right\}, u_{\nu}^{\prime}(\tau)=0$ на С $\Gamma_{2}=\{\angle \tau \in \partial K$, $\left.\left.\frac{-\pi}{2} \leqslant \tau \leqslant \pi\right\}\right\}$, а функция $а$ удовлетворяет условиям 1$), 2$ ).

О корректности задачи (4) с оператором $L_{B}=\square_{B}$ см. [5].

\section{СПИСОК ЛИТЕРАТУРЫ}

[1] Вишик М.И. // Труды ММО. 1952. Т. 1. С. 187-246. [2] Хермандер Л. К теории общих дифференциальных операторов в частных производных. М.: ИЛ, 1959. [3] Ладыженская О.А., Уральцева Н.Н. Линейные и квазилинейные уравнения эллиптического типа. М.: Наука, 1973. [4] Гаевский Х., Грегер К., Захариас К. Нелинейные операторные уравнения и операторные диффференциальные уравнения. М.: Мир, 1978. [5] Burskii V . P. Nonlinear boundary value problems, 7, 1997. IAMM Nat. Acad. Sci. Ukraine, 1997. 